\title{
Tecnologias digitais de informação e comunicação como suporte ao Estágio em Odontologia
}

\author{
Myrna Maria Arcanjo Frota Barros*; Hermínio Borges Neto**; Maria do Socorro de Sousa***; Paulo \\ Goberlanio de Barros Silva****; Cinthia Nara Gadelha Teixeira*****; Maria Eneide Leitão de \\ Almeida $* * * * * *$
}

* Doutora em Clínica Odontológica, Professora do Curso de Graduação em Odontologia, Universidade Federal do Ceará/Campus Sobral

** Doutor, Professor da Faculdade de Educação, Universidade Federal do Ceará

*** Doutora em Saúde Coletiva pela Universidade Federal do Ceará, Coordenadora do OfinArtes

**** Doutor, Professor do Curso de Graduação em Odontologia, Unichristus

***** Doutoranda em Odontologia pela Universidade Federal do Maranhão, Professora do Curso de Graduação em Odontologia, Unichristus

****** Doutora, Professora Associada, Departamento de Clínica Odontológica, Faculdade de Farmácia, Odontologia e Enfermagem, Universidade Federal do Ceará

Recebido em 10/05/2018. Aprovado em 30/06/2019.

\begin{abstract}
RESUMO
O estudo visou aplicar as Tecnologias Digitais de Informação e Comunicação (TDIC) como suporte ao ensino no campo estágio em Odontologia através de um ambiente virtual de ensino (AVE) aberto, que atendesse às necessidades do estágio. Foi elaborado um sistema de avaliação que analisou os diversos aspectos da aprendizagem em campo. Foi implementado um portfólio de atividades como estratégia educativa no AVE e elaboradas categorias e critérios para sua análise. As ferramentas mais usadas no AVE foram o portfólio, o fórum de discussão e o diário de bordo, segundo a opinião dos estudantes. Aproximadamente $80 \%$ dos estudantes e $100 \%$ dos preceptores afirmaram que a inserção do AVE favoreceu o processo de ensino-aprendizagem. As ferramentas digitais criadas foram consideradas facilitadoras da aprendizagem pelos estudantes e preceptores avaliados. As TDIC contribuíam para a melhoria dos cursos de graduação, envolvendo de forma mais intensa alunos e professores, integrando-os às estratégias de comunicação, muitas vezes já tão utilizadas em contextos diversos fora da universidade. Foi possível caracterizar os estudantes analisados como sendo a maioria do sexo feminino, solteiros e sem filhos. Além disso, a maioria dos discentes somente estuda. Os estudantes avaliaram como positivo o desempenho dos preceptores. Não foram relatadas dificuldades vivenciadas pelos estudantes e preceptores com a utilização do AVE. O uso dessas tecnologias focou nos componentes curriculares do curso envolvidos, priorizando o acesso síncrono e assíncrono aos conteúdos, a autonomia dos discentes e a disseminação de uma cultura digital. A adequação das metodologias de ensino, permitiu melhorias no desempenho acadêmico dos alunos, professores e preceptores e estimulou o processo de educação permanente, facilitando o ensino-aprendizagem no Estágio em Odontologia.

Descritores: Educação em Saúde. Avaliação Educacional. Educação a distância.
\end{abstract}




\section{INTRODUÇÃO}

Desde os anos 1950 buscava-se ofertar, nos cursos da área de saúde, experiências que proporcionassem a integração ensino-serviço. Entretanto, esse tema, apesar de ser debatido há mais de meio século, ainda é atual, pois o ensino e os serviços de saúde são dinâmicos, estando sempre em processo de mudança ${ }^{1}$.

O curso de Odontologia tem como um de seus maiores desafios a consolidação do estágio supervisionado como uma estratégia de real impacto na transformação da formação profissional, no contexto de uma estrutura curricular abrangente, que contemple tanto a prática clínica de disciplinas específicas quanto experiências que visam à integração ensino-serviços-comunidade ${ }^{1-3}$.

Essa relação ensino-serviço é complexa, dependendo de muitos fatores locais. De um lado a universidade, que dispõe de poucos professores dedicados aos estágios, sendo o número de estudantes muitas vezes incompatível com o número de professores, resultando numa supervisão inadequada; do outro, a rede de serviços, que nem sempre pode acomodar todos os estagiários em um só local, dificultando a adaptação dos horários entre os preceptores de campo, alunos e a supervisão docente. Além disso, muitas vezes falta clareza na definição das atribuições do professor e do preceptor para o trabalho em equipe e avaliação das atividades desenvolvidas $^{2-3}$.

Nessa perspectiva, o presente estudo buscou implantar a utilização de Tecnologias Digitais de Informação e Comunicação (TDIC) no processo de ensino-aprendizagem no Estágio em Serviço do SUS I do curso de Odontologia da Universidade Federal do Ceará (UFC). Assim, foi utilizado um ambiente virtual de ensino (AVE) como prática pedagógica e social, como linguagem para representação do conhecimento e, portanto, estruturante no currículo.

Nesse contexto, foi implementado no AVE, um portfólio educacional como objeto de estudo para os acadêmicos. O portfólio é entendido como instrumento facilitador da construção e reconstrução do conhecimento, permitindo a reflexão do aluno sobre a realidade local, identificando os problemas e analisando-os criticamente. A busca de conhecimento, a criatividade e a produção escrita são incentivadas para que o aluno trilhe seu próprio percurso formativo acompanhado pelo professor, que avaliará esse caminhar ${ }^{4}$.

Os AVE também são muito utilizados como meios de avaliação, pois concentram a atenção de todos (dos estudantes de um mesmo grupo, dos professores e dos preceptores), tornando importante os trabalhos dos estagiários e facilitando a troca de experiências entre os grupos. Apesar da ampla utilização de AVE em diversas áreas do conhecimento, ainda é raro o uso dessa metodologia ativa nos cursos de Odontologia. Na literatura também é possível observar a inexistência dessa experiência em estágios dos cursos de Odontologia.

Os objetivos desse estudo foram criar um AVE e avaliar o processo de ensino-aprendizagem no estágio curricular do Curso de Odontologia, além de caracterizar o perfil sociodemográfico e de formação dos discentes; analisar se houve benefícios ao processo de ensino-aprendizagem com o uso do AVE; avaliar o desempenho dos preceptores, segundo a opinião dos estagiários, e identificar possíveis dificuldades vivenciadas pelos estudantes e preceptores com a utilização do AVE durante o processo de ensino-aprendizagem.

\section{METODOLOGIA}

A pesquisa foi aprovada pelo Comitê de Ética em Pesquisa da UFC (protocolo 542.042, CAAE: 18207613.1.0000.5054) e os sujeitos que concordaram em participar assinaram o Termo de Consentimento Livre e Esclarecido.

Trata-se de um estudo descritivo, transversal com uma abordagem quantitativa, realizado no 
Curso de Odontologia da UFC. A coleta de dados foi realizada por único pesquisador, por meio de questionário estruturado, dividido em cinco categorias de análise: perfil sociodemográfico, perfil de formação, avaliação do AVE TelEduc, avaliação do processo de ensino-aprendizagem e avaliação do preceptor. $\mathrm{O}$ instrumento foi distribuído aos estudantes e preceptores no último dia do estágio, ocasião em que foi realizada a apresentação de um seminário final sobre as atividades desenvolvidas em cada campo.

A validação do questionário foi realizada em teste piloto com 10 estudantes da disciplina, a fim de analisar a compreensão do instrumento como um todo e de cada item isoladamente. Os estudantes que participaram o teste piloto foram excluídos da amostra final. Foi definido como campo de estudo o Estágio em Serviços do SUS I (ESSUS I), realizado em 04 Unidades de Atenção Primária à Saúde (UAPS) no $9^{\circ}$ semestre do Curso de Odontologia da UFC, contando com 03 professores supervisores, 04 preceptores de campo - cirurgiões dentistas do serviço de saúde, com 40 vagas por semestre e carga horária de três créditos, equivalendo a 48 horas semanais de atividades em campo.

Participaram da pesquisa os estudantes e preceptores em atividade em dois semestres letivos. Os alunos deveriam estar regularmente matriculados no ESSUS I e terem participado das atividades a distância no TeleEduc para serem incluídos na amostra.

Implantação de um Ambiente Virtual de Aprendizagem para uso universitário, que utilize as tecnologias da Internet no processo educacional e atenda as peculiaridades do Estágio

O TelEduc é um software livre e aberto de ensino a distância pelo qual se pode realizar cursos via Internet, desenvolvido pelo Núcleo de Informática Aplicada à Educação (NIED) da Universidade Estadual de Campinas (UNICAMP), disponível por meio da UFC Virtual, pela Universidade Aberta do Brasil. Seu desenvolvimento se deu desde 1997 de forma participativa, ou seja, todas as suas ferramentas foram idealizadas e projetadas a partir das necessidades dos usuários, além de possuir suporte à múltiplas línguas de forma a atender a demanda de uso internacional do ambiente. Possui características que o diferencia dos demais ambientes para educação a distância disponíveis no mercado, como a facilidade de uso por pessoas não especialistas em computação e um conjunto conciso de funcionalidades 5 .

\section{Gestão Pedagógica e gerenciamento do AVE}

Para a execução do projeto foram necessárias reuniões e oficinas de capacitação pedagógica de professores e preceptores com uma pedagoga, além da capacitação de professores, preceptores e estudantes para o uso do AVE.

O apoio técnico para o gerenciamento do AVE foi realizado pelo Laboratório de Pesquisa Multimeios da Faculdade de Educação/UFC, que possui um sólido patrimônio em conhecimento e tecnologia e conta com o trabalho de monitores para o esclarecimento de dúvidas em sistema online.

\section{Sistema de avaliação}

Buscou-se empregar no estágio os princípios da avaliação formativa, que pode ser entendida como "uma prática de avaliação contínua que tem como objetivo principal melhorar as aprendizagens em curso, contribuindo para o acompanhamento e orientação dos alunos durante todo seu processo de formação"6. Esta modalidade de avaliação fornece informações importantes acerca do processo de ensino-aprendizagem: ao professor, que será informado dos efeitos reais de suas ações; e ao estudante, que terá oportunidade de conhecer suas dificuldades e, possivelmente, reconhecer e corrigir seus próprios erros. 
Foi implantado um sistema de avaliação no ESSUS I que possibilitou a intervenção nos diversos aspectos da aprendizagem, sendo a nota final dos estudantes composta pelos seguintes critérios e categorias de análise: assiduidade e participação nos encontros presenciais, assiduidade $\mathrm{e}$ participação nas atividades a distância e nota do portfólio.

A participação nos encontros a distância foi avaliada por meio dos resultados gerados pelas ferramentas InterMap e Acessos, que analisaram o quantitativo dos registros das interações. $\mathrm{O}$ InterMap mapeia a interação e a participação dos integrantes de um curso $^{7}$ e a ferramenta Acessos gera relatórios sobre os acessos dos usuários ao curso e a cada uma das ferramentas do TelEduc ${ }^{5}$. A avaliação contínua a distância poderia ser realizada por meio da análise dos registros das participações no curso e tinha especial importância no contexto da Educação a Distância, por possibilitar a percepção do comportamento do estudante e auxiliar a identificação de problemas ${ }^{8}$.
Um sistema também foi implantado para avaliar o portfólio educacional, contendo categorias e critérios de análise baseados nas dimensões cognitiva, produtiva, atitudinal e relacional que os estudantes deveriam desenvolver ao longo do estágio, (organização do conhecimento, competência para avaliar sua própria atuação, elaboração ordenada de conceitos e desenvolvimento de competências e habilidades, dentre as quais destacou-se a prática reflexiva).

O Sistema de Avaliação foi norteado pelos princípios da avaliação formativa e pela definição de competências, que é o conjunto de conhecimentos, habilidades, atitudes e comportamentos manifestados no desempenho de determinadas atividades por meio de ações observáveis pelos demais participantes do processo $^{8}$. O quadro 1 apresenta como foram catalogadas as notas finais dos estudantes e o quadro 2 contém os critérios e categorias de análise do portfólio.

Quadro 1. Composição da nota final dos estudantes regularmente matriculados no Estágio em Serviços do SUS I, com o uso de TDIC

\begin{tabular}{|c|c|c|c|c|}
\hline$\%$ Frequência & $\begin{array}{c}\text { Assiduidade e participação } \\
\text { nos encontros presenciais } \\
(0-30 \text { pontos })\end{array}$ & $\begin{array}{c}\text { Assiduidade e participação } \\
\text { nas atividades a distância } \\
(\mathbf{0 - 2 0} \text { pontos })\end{array}$ & $\begin{array}{c}\text { Portfólio } \\
(\mathbf{0 - 5 0} \text { pontos })\end{array}$ & $\begin{array}{c}\text { Nota final } \\
(\mathbf{1 0 0} \text { pontos })\end{array}$ \\
\hline
\end{tabular}

Quadro 2. Composição da nota final dos portfólios dos estudantes regularmente matriculados no Estágio em Serviços do SUS I

\begin{tabular}{|c|c|c|c|c|}
\hline $\begin{array}{c}\text { Organizar o } \\
\text { saber do } \\
\text { estudante } \\
(0-10 \text { pontos })\end{array}$ & $\begin{array}{c}\text { Aprimorar competências/ } \\
\text { habilidades: linguística, } \\
\text { observação participação, } \\
\text { prática reflexiva e outras } \\
(0-10 \text { pontos })\end{array}$ & $\begin{array}{c}\text { Propiciar aos participantes } \\
\text { desenvolver competência } \\
\text { para avaliar sua atuação }\end{array}$ & $\begin{array}{c}\text { Facilitar } \\
\text { elaboração } \\
\text { ordenada de } \\
\text { conceitos }\end{array}$ & $\begin{array}{c}\text { Total } \\
(50 \\
\text { pontos })\end{array}$ \\
& $(0-20$ pontos $)$ & \\
\hline
\end{tabular}

\section{Análise Estatística}

Os dados foram tabulados no Microsoft Excel e exportados para o software estatístico Statistical Packcage for the Social Sciences
(SPSS), versão 17.0, no qual todas as análises foram realizadas considerando nível de confiança de $95 \%$.

Foram calculadas as frequências absolutas e 
percentuais dos dados apresentados, os quais foram analisados por meio dos testes exato de Fisher ou qui-quadrado, a depender da indicação. O número de filhos e a idade dos entrevistados foram analisados por meio do teste $\mathrm{t}$ de Student (dados paramétricos, normalidade verificada pelo teste de Kolmogorov-Smirnov).

\section{RESULTADOS}

\section{Perfil sociodemográfico}

A população do estudo foi de 72 estudantes (95\%) de um total de 76 regularmente matriculados no estágio e 4 preceptores (100\%) envolvidos.

O perfil dos estudantes, foi idade média de $23,5 \pm 1,7$ anos, sendo $66,7 \% \quad(\mathrm{n}=48)$ do sexo feminino, 95,8\% ( $\mathrm{n}=69)$ solteiros, 97,2\% ( $\mathrm{n}=70)$ não possuíam filhos e 76,4\% (n=55) moravam com os pais. A maioria dos discentes $(95,8 \%-n=69)$ relatou somente estudar. Em relação aos preceptores de campo entrevistados, a média de idade foi de aproximadamente 35 anos, sendo todos casados e do sexo feminino.

Constatou-se que $97,2 \%$ dos estudantes analisados possuíam computador em casa para realizar as atividades a distância. Entretanto, aproximadamente $48,6 \%$ afirmaram que esse computador não é de uso individual, ou seja, é compartilhado com o restante da família.

Em relação ao tipo de internet utilizada, $84,7 \%$ dos estudantes faziam uso da banda larga, facilitando a realização das atividades solicitadas no TelEduc. Quanto ao acesso à internet para realização destas atividades, $91,7 \%$ dos pesquisados afirmaram utilizar a internet em casa e apenas, 4,2\% afirmam acessar a internet na faculdade. $\mathrm{O}$ cruzamento da variável ter computador em casa, com tipo de internet e local de acesso permitiu comprovar estatisticamente que os estudantes que têm computador acessam a internet através de banda larga e com mais frequência em casa, o que favorece o uso de metodologias de ensino aprendizagem a distância (tabela 1). Todos os preceptores que fizeram parte do estágio possuem computador em casa, com acesso à internet banda larga.

Um dos indicadores de qualidade de uma disciplina com suporte a distância é a infraestrutura, onde se destacam os laboratórios de informática. Nesse sentido, a Faculdade de Farmácia, Odontologia e Enfermagem da UFC disponibiliza laboratório de informática com 24 computadores e acesso à internet. Já o curso de Odontologia da instituição disponibiliza uma sala de apoio técnico com sete computadores com acesso à internet, uma impressora e duas mesas para reunião, que forneciam o suporte pedagógico. Havia também o apoio técnico para o gerenciamento do AVE, que era feito pelo Laboratório de Pesquisa Multimeios da Faculdade de Educação/UFC.

\section{Perfil da formação}

A maior parte dos estudantes entrevistados $(95,8 \%)$ participava ou já tinha participado de algum projeto de extensão universitária, mostrando diferença estatisticamente significante em relação às outras atividades por eles desempenhadas, como monitoria, pesquisa, estágio não obrigatório, cursos extracurriculares e a distância.

Quanto ao perfil de formação da preceptoria os resultados mostraram que $50 \%$ $(\mathrm{n}=2)$ dos preceptores já realizaram algum curso a distância. Porém, somente $25 \% \quad(n=1)$ dos preceptores de campo tinha experiência com preceptoria à distância, dado que mostrou a importância da capacitação antes do início das atividades no AVE.

\section{O Ambiente Virtual de Ensino}

A ferramenta do TelEduc que mais contribuiu para a aprendizagem segundo os estudantes foi o portfólio, diferindo 
significantemente das demais. Porém, na opinião dos preceptores, foi o fórum de discussão que mais contribuiu para o processo de ensinoaprendizagem dos discentes $(75 \%, \mathrm{n}=3)$.

\section{Avaliação do processo de ensino-aprendizagem}

A maioria dos estudantes abordados considerou o TelEduc favorecedor da aprendizagem $(83,6 \%, \mathrm{n}=56)$ (tabela 2).

Tabela 1. Associação entre as variáveis ter computador em casa, segundo ter internet em casa, tipo de internet e local de acesso dos estudantes do ESSUS I no Curso de Odontologia

\begin{tabular}{lccc}
\hline & \multicolumn{2}{c}{ Tem computador em casa } & \\
Não & Sim & p-Valor \\
\hline Tem internet & $1(50,0 \%)$ & $2(2,9 \%)$ & 0,084 \\
$\quad$ Não & $1(50,0 \%)$ & $66(97,1 \%)$ & \\
$\quad$ Sim & & & \\
Tipo de internet & - & $61(98,4 \%)^{*}$ & 0,032 \\
$\quad$ Banda Larga & $1(100,0 \%)$ & $1(1,6 \%)$ & \\
3G & & & \\
Onde mais acessa & $1(50,0 \%)$ & $65(92,9 \%)$ & $<0,001$ \\
Em casa & $1(50,0 \%)$ & - & \\
Em Lan House & - & $1(1,4 \%)$ & \\
Na casa de familiares & - & $3(4,3 \%)$ & \\
Na faculdade & - & $1(1,4 \%)$ & \\
Outros & & & \\
\hline *p $<0,05$ - Teste Exato de Fisher ou Qui-quadrado &
\end{tabular}

Tabela 2. Associação entre as variáveis opinião dos estudantes do ESSUS I do Curso de Odontologia sobre o processo de ensino-aprendizagem e o TelEduc como favorecedor da aprendizagem

\begin{tabular}{|c|c|c|c|}
\hline \multicolumn{4}{|c|}{ Considera o TELEDUC favorecedor aprendizagem } \\
\hline & Não & Sim & p-Valor \\
\hline \multicolumn{4}{|l|}{ As orientações foram claras } \\
\hline Concordo plenamente & $3(27,3 \%)$ & $31(56,4 \%)^{*}$ & 0.026 \\
\hline Concordo parcialmente & $7(63,6 \%)^{*}$ & $24(43,6 \%)$ & \\
\hline Discordo & $1(9,1 \%)^{*}$ & - & \\
\hline \multicolumn{4}{|l|}{ O tempo foi suficiente } \\
\hline Concordo plenamente & $5(45,5 \%)$ & $39(70,9 \%)^{*}$ & 0.022 \\
\hline Concordo parcialmente & $3(27,3 \%)$ & $14(25,5 \%)$ & \\
\hline Discordo & $3(27,3 \%)^{*}$ & $2(3,6 \%)$ & \\
\hline \multicolumn{4}{|c|}{ A metodologia contribuiu para a aprendizagem } \\
\hline Concordo plenamente & - & $26(47,3 \%)^{*}$ & $<0.001$ \\
\hline Concordo parcialmente & $7(63,6 \%)$ & $29(52,7 \%)$ & \\
\hline Discordo & $4(36,4 \%)^{*}$ & - & \\
\hline \multicolumn{4}{|c|}{ As estratégias facilitaram a aprendizagem } \\
\hline Concordo plenamente & - & $30(54,5 \%)^{*}$ & $<0.001$ \\
\hline Concordo parcialmente & $8(72,7 \%)^{*}$ & $25(45,5 \%)$ & \\
\hline Discordo & $3(27,3 \%) *$ & - & \\
\hline
\end{tabular}


Todos os preceptores afirmaram que as estratégias de avaliação facilitaram a compreensão dos assuntos, e consideram que o TelEduc favoreceu o processo de ensino-aprendizagem.

\section{Avaliação do preceptor}

Os estudantes também tiveram a oportunidade de avaliar a preceptoria de campo. Segundo a maioria dos estagiários $(43,7 \%, \mathrm{n}=31)$, os preceptores sempre estimularam as atividades no AVE TelEduc, tendo esse resultado relevância estatística.

Ainda sobre a avaliação da preceptoria, os estudantes concordaram que os preceptores esclareceram dúvidas $(59,2 \%, n=49)$ e sempre contribuíram para a compreensão dos conteúdos (54,9\%\%, $\mathrm{n}=39)$, resultados com diferenças estatísticas sobre as demais possíveis respostas.

Os preceptores também tiveram a oportunidade de se autoavaliar: $50 \% \quad(\mathrm{n}=2)$ consideram que frequentemente estimularam a participação no TelEduc, 25\% (n=1) afirmaram que sempre estimularam os discentes, tendo igual percentual afirmado que essa motivação ocorreu às vezes. Porém, a maioria dos preceptores $(75 \%$, $\mathrm{n}=3$ ) considerou que sempre esclareceu as dúvidas dos estagiários.

\section{DISCUSSÃO}

A maioria dos discentes desse estudo era do sexo feminino, realidade também já observada em algumas pesquisas ${ }^{9,10}$ que comprovaram a predominância do sexo feminino nos cursos de Odontologia. Eram em sua maioria solteiros, não possuíam filhos e moravam com os pais, resultados semelhantes já tinham sido encontrados ${ }^{9}$.

É fundamental que a instituição possua laboratório de informática com acesso a internet banda larga de qualidade, pois o sucesso de educação a distância para disciplinas semipresenciais está na disponibilidade de infraestrutura para os discentes que não dispõe de acesso à internet fora da universidade. Assim, o acompanhamento da disciplina e a realização de atividades propostas podem ser realizados sem prejuízos, pois os estudantes devem ter livre acesso para consultar a internet e compatibilidade entre a quantidade de equipamentos e o número de estudantes atendidos, para que se possa garantir a qualidade ${ }^{11,12}$.

O perfil de formação dos discentes e preceptores mostrou que a educação a distância ainda não tinha sido vivenciada por todos. Nos últimos anos, ocorreu um aumento exponencial do número de cursos oferecidos e matrículas nesta modalidade. Porém, ainda é possível perceber a resistência de muitos cursos de educação superior em aderir a essa nova modalidade de ensino, visto que muitos autores questionam sua qualidade e efetividade, apesar de pesquisas recentes concluírem que a modalidade de ensino a distância pode alcançar níveis de qualidade equivalentes ou superiores à modalidade presencial ${ }^{13,14,20}$.

Os discentes acreditaram ser o portfólio o instrumento que mais contribuiu para a sua aprendizagem, atendendo à necessidade de aprofundar o conhecimento sobre a relação ensinoaprendizagem, assegurando aos estudantes e professores uma compreensão maior do que foi ensinado e, desse modo, índices mais elevados de qualidade $^{15}$.

O TelEduc foi considerado pelos estudantes e preceptores um favorecedor da aprendizagem. Esse resultado foi importante, pois o desenvolvimento da autonomia e da auto-organização é visto como um dos ganhos para a formação dos alunos que participam de cursos na modalidade semipresencial, visto que as atividades a distância demandam tais comportamentos, pois há a flexibilização da aprendizagem em relação ao tempo e espaço ${ }^{11}$.

Os ambientes virtuais de aprendizagem possibilitam o acompanhamento da frequência e da produção de cada estudante, uma vez que consistem 
em uma grande base de dados que armazena a frequência e assiduidade (data e hora de acessos ao ambiente, data e hora de acessos a cada uma das ferramentas disponíveis no ambiente), trabalhos publicados, tarefas realizadas e também as mensagens trocadas entre os participantes de um curso $^{16,17}$.

Dessa forma, o uso dos ambientes virtuais oferece novas maneiras de atuar no ensino, praticando a modalidade do ensino a distância auxiliar em disciplinas presenciais, contribuindo no ensino-aprendizagem e facilitando a comunicação entre professores e estudantes.

A percepção dos estagiários quanto ao preceptor como estimulador da atividade do AVE foi relevante, pois o efetivo envolvimento do preceptor é de fundamental importância, devendo ser um motivador e incentivador do processo de ensinoaprendizagem. Sem a participação e estímulo do preceptor o sucesso de um curso a distância diminui consideravelmente $^{18,21}$. É necessário um processo de capacitação para que os preceptores envolvidos nos estágios possam perceber sua importância em estimular seus estagiários na participação das atividades a distância.

\section{CONCLUSÕES}

As ferramentas digitais criadas foram consideradas facilitadoras da aprendizagem pelos estudantes e preceptores avaliados. As TDIC contribuíam para a melhoria dos cursos de graduação, envolvendo de forma mais intensa alunos e professores, integrando-os às estratégias de comunicação, muitas vezes já tão utilizadas em contextos diversos fora da universidade.

Foi possível caracterizar os estudantes analisados como sendo a maioria do sexo feminino, solteiros e sem filhos. Além disso, a maioria dos discentes somente estuda.

Os estudantes avaliaram como positivo o desempenho dos preceptores. Não foram relatadas dificuldades vivenciadas pelos estudantes e preceptores com a utilização do AVE.

$\mathrm{O}$ uso dessas tecnologias focou nos componentes curriculares do curso envolvidos, priorizando o acesso síncrono e assíncrono aos conteúdos, a autonomia dos discentes e a disseminação de uma cultura digital. A adequação das metodologias de ensino, permitiu melhorias no desempenho acadêmico dos alunos, professores e preceptores e estimulou o processo de educação permanente, facilitando o ensino-aprendizagem no Estágio em Odontologia.

\section{ABSTRACT \\ Digital information and communication technologies as a support to the Internship in Dentistry}

This study aimed to apply the Digital Information and Communication Technologies (DICT) as a support to the teaching in the training ground of Dentistry through an open virtual teaching environment (VTE), that meets the needs of the internship. An evaluation system has been developed that has analyzed many aspects of field learning. A portfolio of activities was implemented as an educational strategy in the VTE and categories and criteria for its analysis were elaborated. The most used tools in the VTE were the portfolio, the discussion forum and the logbook, according to the students' opinion. Approximately $80 \%$ of the students and $100 \%$ of the tutors stated that the insertion of the VTE favored the teachinglearning process. The digital tools created were considered to be learning facilitators by the evaluated students and tutors. DICT has contributed to the improvement of undergraduate courses, involving students and teachers more intensely, integrating them into communication strategies, often already used in diverse contexts outside the university. It was possible to characterize the analyzed students as being the majority female, single and childless. In addition, most students only study. The students evaluated the performance of the tutors as positive. No difficulties experienced by students and tutors with the use of the VTE were reported. The use of these technologies focused on the curricular components 
of the course involved, prioritizing synchronous and asynchronous access to content, student autonomy and the dissemination of a digital culture. The adequacy of teaching methodologies allowed for improvements in the academic performance of students, teachers and tutors and stimulated the process of permanent education, facilitating teaching-learning in the Internship in Dentistry.

Descriptors: Health Education. Educational Measurement. Education, Distance.

\section{AGRADECIMENTOS}

Ao Programa Pró-Ensino da Saúde da Coordenação de Aperfeiçoamento de Pessoal de Nível Superior (Capes) pela concessão da bolsa de mestrado.

Ao Laboratório de Multimeios da Universidade Federal do Ceará pelo apoio técnico.

\section{REFERÊNCIAS}

1. Werneck MAF, Senna MIB, Drumond MM, Lucas SD. Nem tudo é estágio: contribuições para o debate. Ciênc Saúde Coletiva. 2010; 15 (1): 221-31.

2. Santos KT, Batista RJ, Bitencourt CTF, Araújo RP, Carvalho RB. Percepção discente sobre a influência de estágio extramuro na formação acadêmica odontológica. Rev Odontol UNESP 2013; 42(6): 420-25.

3. Toassi RFC, Davoglio RS, Lemos VMA. Integração ensino-serviço-comunidade: o estágio na atenção básica da graduação em odontologia. Educ Rev 2012; 28(4):232-42.

4. Frota MMA, Menezes LMB, Alencar $\mathrm{CH}$, Jorge LS, Almeida MEL. O portfólio como estratégia facilitadora do processo de ensinoaprendizagem para a formação em odontologia. Adequação de metodologias de ensino utilizando o ambiente virtual de aprendizagem. Rev ABENO 2011; 11(1): 23-28.

5. Rocha VH. O ambiente TelEduc para Educação à Distância baseada na Web: Princípios, Funcionalidades e Perspectivas de desenvolvimento. In: Moraes MC, organizadores. Educação à Distância: Fundamentos e Práticas. Campinas: Unicamp/Nied; 2002. p. 197-212.

6. Perrenoud P. Avaliação: da excelência à regulação das aprendizagens entre duas lógicas. Porto Alegre: Artes Médicas; 1999.

7. Romani LAS. Intermap: Ferramenta para Visualização da Interação em Ambientes de Educação a Distância na Web [dissertação]. Unicamp; 2000.

8. Otsuka JL, Ferreira TB, Lachi RL Rocha HV. Um modelo de suporte à avaliação formativa no ambiente TelEduc. RBIE 2003; 11(2):1-12.

9. Carvalho ACP. O "milagre" da multiplicação dos cursos. Rev Assoc Paul Cir Dent. 1997; 51(4): 310-18.

10. Galassi MS. Expectativas do cirurgiãodentista em relação ao mercado de trabalho. Rev Assoc Paul Cir Dent. 2004; 58(1): 6770.

11. Bertolin JCG, Marchi ACB. Instrumentos para avaliar disciplinas da modalidade semipresencial: uma proposta baseada em sistemas de indicadores. Avaliação (Campinas) 2010; 15(3): 131-146.

12. Brasil. Ministério da Educação. Referenciais de Qualidade para Educação Superior a Distância. Secretaria de Educação a Distância. Brasília, 2007; ago.

13. ABRAEAD. Anuário Brasileiro Estatístico de Educação Aberta e a Distância. $4^{a}$ Edição. São Paulo: Instituto Monitor 2008.

14. INEP/MEC. Na Medida - Boletim de Estudos Educacionais do Inep. Ano 1, número 3 2009; set. 
15. Vieira VMO. Portfólio: uma proposta de avaliação como reconstrução do processo de aprendizagem. Psic Esc Educ. 2002; 6(2):149-53.

16. Bassani OS, Bearh PA. Análise das interações em ambientes virtuais de aprendizagem: uma possibilidade para avaliação da aprendizagem em EaD. RENOTE 2006; 4(1): 1-10.

17. Schlemmer E, Saccol AZ, Garrido S. Um modelo sistêmico de avaliação de softwares para educação a distância como apoio à gestão de EaD. REGE USP 2007;14(1):77-91.

18. Barbosa, MFSO; Rezende, F. A prática dos tutores em um programa de formação pedagógica à distância: avanços e desafios. Interface (Botucatu) 2006; 10(20):473-86.

19. Ariana $A$, et al. Integration of Traditional and E-Learning Methods to Improve Learning Outcomes for Dental Students in Histopathology. J Dent Educ. 2016;
80(9):1140-8.

20. Olmsted JL. Direct assessment as a measure of institutional effectiveness in a dental hygiene distance education program. J Dent Educ 2014; 78(10):1460-7.

21. dos Santos CM, Bulgarelli PT, Frichembruder K, Colvara BC, Hugo FN.. Avaliação da qualidade de aprendizagem no ambiente virtual (Moodle) em saúde bucal, na perspectiva dos discentes. Rev ABENO 2018; 18(1): 116-23.

\section{Correspondência para:}

Myrna Maria Arcanjo Frota Barros e-mail: myrnarcanjo@ hotmail.com

Rua General Silva Júnior, 640/1105 Torre 2 Bairro de Fátima

60.411-200 Fortaleza/CE 